\title{
STABILITY AND INSTABILITY OF HIERARCHICAL TRIPLE
} SYSTEMS

\author{
L.KISELEVA ${ }^{1}$, J.ANOSOVA $^{2}$,P.EGGLETON ${ }^{1}$, J.COLIN $^{3}$, V.ORLOV $^{4}$ \\ ${ }^{1}$ Institute of Astronomy, Madingley Rd., Cambridge \\ 2 National Astronomical Observatory, Tokyo 181, Japan \\ 3 Observatoire de Bordeaux, Floirac, France \\ 4 Astronomical Institute,St. Peterburgh University, \\ St. Petersburg 198904, Russia
}

We computed the dynamical evolution of hierarchical triple stars in which both orbits are initially circular, and determine the lower limit to the ratio of periods (outer/inner) for which there is dynamical stability. We found for some mass ratios resonance-like behaviour that occurs in a limited range of initial period ratio. Some resonances are 'disruptive'; that is, for a small range of initial period ratio we find that the system is not able to settle down to a quasi-steady hierarchical state, but instead disrupts. However, below as well as above this disruptive range there are considerable ranges of initial period ratio where the hierarchical state appears to be stable, at least for the length of integration time we took which was sometimes as much as 10,000 outer orbits. The mass ratios are identified for which different types of unstable behaviour, such as an escape of the distant body without exchange, many exchanges in the limited space without escape, formation of new long-live hierarchy, or an escape of one body after a few exchanges, occur for ratios of periods slightly below the limit of stability. We discuss the relevance of the above behaviour to observed close triples, the closest of which is $\lambda$ Tau (period ratio 8.3).

In a very small region of our three-dimensional parameter space we have discovered a family of periodic orbits. The masses involved are approximately $1.0+0.016$ for the inner binary, and 0.4 for the third body. The lightest body makes alternately two small and two large revolutions about the heaviest body, viewed in the frame where the two heaviest bodies are at rest. This pattern persisted for at least several thousand revolutions. 